\title{
Historical perspectives in the assessment of cardiovascular structure and function
}

\author{
Keith P. George ${ }^{1}$. Daniel J. Green ${ }^{2}$
}

Received: 2 March 2018 / Accepted: 26 March 2018 / Published online: 19 April 2018

c) Springer-Verlag GmbH Germany, part of Springer Nature 2018

There is a long history of novel techniques and observations having a profound impact in cardiovascular physiology and medicine. Indeed, Harvey's De Motu Cordis is considered by some historians to be the founding document for what became the modern scientific method. Harvey overthrew hundreds of years of received Galenic wisdom by carefully observing and calculating physiological function, then proving his suppositions to be correct in simple, elegant but revolutionary experiments. His approach exemplified the enlightenment precept "trust what you can see ...., then see what you can trust". In this vein, the European Journal of Applied Physiology has recently supported and developed a number of review papers on the theme of "Historical Perspectives" in applied human physiology. These brief reviews are aimed at explaining how techniques evolved, or were disrupted, and the relative strengths and weaknesses of the approaches that emerged.

Multiple aspects of cardiovascular structure and function fall within the general remit of the journal and have been of significant interest to scientists, clinicians and the general public. For example, we know that optimal functioning of the cardiovascular system is fundamental to supporting high level sporting performance. Conversely, we know that diseases of the cardiovascular system still account for a significant proportion of deaths around the globe despite the fact that exercise and physical activity are potent positive mediators of primary and secondary cardiovascular risk. The cardiovascular system is not only very responsive to acute and chronic stress but also highly vulnerable to disuse and

Communicated by Michael Lindinger.

Keith P. George

K.George@1jmu.ac.uk

1 Research Institute for Sport and Exercise Sciences, Liverpool John Moores University, Liverpool L3 3AF, UK

2 Sport Science, Exercise and Health, School of Human Sciences, University of Western Australia, Perth 6009, Australia disease and this combination of factors has prompted multiple areas of study.

Given the importance of the cardiovascular system in both health and disease, it is no surprise that there is a long history of innovation in measurement and assessment that have helped underpin basic and applied studies of the human heart and vasculature. Some elements of cardiovascular structure and function are relatively easy to assess in humans; such as heart rate; but other crucial facets are more complicated to measure accurately using non-invasive techniques; such as muscle blood flow. It is, of course, vital that our invasive or non-invasive tools to measure cardiovascular structure and function are both valid and reliable, as these parameters will underpin their utility in human physiology studies. With these issues in mind, this series will cover a historical timeline of measurement tool development, evaluate validity and reliability, as well as discuss the utility and limitations of each tool when interrogating various aspects of human health and physiology.

The first paper in the current series is a historical perspective of methods for the determination of skeletal muscle blood flow (Gliemann et al. 2018). The authors skilfully weave critical commentary about the accuracy and value of different tools in a general timeline from the first measurements of venous outflow in the nineteenth century (Sadler 1869) to more recent technological developments like contrast-enhanced ultrasound (Coggins et al. 2001). As well as the historical element of the narrative the paper clearly links specific tools to their application in a range of physiological and health questions. Finally, the authors discuss the current limits of our technical options and knowledge that links to directions for on-going research and product development.

Later papers in this series will evaluate measurement options associated with other discrete elements of the cardiovascular system. For example, the historical development of assessment tools applied to cardiac structure and function, cerebral blood flow and skin blood flow will be discussed. The development and use of conduit artery functional 
indices in both health and disease will be addressed. Whilst there will obviously be some technical overlap between papers; the use of Doppler ultrasound being a case in point; in all cases the specific and unique application of techniques will be carefully reviewed.

Like all of the "Historical Perspective" reviews, the papers in this series on cardiovascular structure and function should serve as; (1) valuable educational tools to remind us all of where key developments occurred and how they have been adopted by scientists and clinicians, (2) a thorough and systematic comparison of the strengths and weaknesses of measurement options available to current scientists studying the human cardiovascular system, and (3) a primer for ongoing technical and research developments that are important to allow our field to make progress in the twenty-first century and continue to answer key scientific and clinical questions.

Author contributions Both Keith George and Daniel Green contributed equally to this editorial.

\section{References}

Coggins M, Lindner J, Rattigan S, et sl (2001) Physiological hyperinsulinemia enhances human skeletal muscle perfusion by capillary recruitment. Diabetes 50:2682-2690

Gliemann L, Mortensen SP, Hellsten Y (2018) Methods for the determination of skeletal muscles blood flow: development, strengths and limitations. Eur J Appl Physiol. https://doi.org/10.1007/s0042 1-018-3859-2

Sadler W (1869) Arbeiten aus der phys, 12 edn. Anstalt zu, Leipzig 